\title{
Magnetic dipole source TEM all-time apparent resistivity one- dimensional forward modeling and optimization calculation
}

\author{
Zhongyuan $\operatorname{Sun}^{1}$ and Qingxin Meng ${ }^{1}$ \\ ${ }^{1}$ College of Earth Sciences, Hebei GEO University, No.126, Huai'an Road, Shijiazhuang, Hebei Prvince, China
}

\begin{abstract}
Carrying out the forward modeling and one-dimensional apparent resistivity optimization calculation of TEM(transient electromagnetic method), theoretically verifies the the possibility that obtains the apparent resistivity in the full time domain under the condition of multiple offsets. By calculating the second derivative, Halley's method can accelerate the iteration and make the function converge. The moment, offset and apparent resistivity are controlled by variable method. Halley's method is used to iterate the moment, offset and apparent resistivity for completing the optimization calculation. In this paper, a conclusion will be finally drawn that the multi-offset TEM can get the apparent resistivity in the full time domain.
\end{abstract}

\section{Introduction}

Transient electromagnetic method(TEM) is a widely used electromagnetic exploration method in time domain ${ }^{[1]}$. Predecessors mainly conducted plenty of indepth studies on the working mode of the central loop or overlapping coils of the magnetic source TEM, namely, the magnetic source TEM with zero offset, and found that increasing the multi-turn small transmitting coil can improve the inversion depth, and conversely decreasing it can improve the inversion accuracy ${ }^{[2]}$.

The whole time domain is often divided into three parts: the early, middle and late time domain. The early time domain corresponds to shallow underground depth and is often used for shallow hydrogeological, environmental ${ }^{[3]}$ and structural exploration ${ }^{[4]}$.Plotnikov conducted numerical experiments using training software and found that shallow depth meant that the response curve could not adequately reflect the nonuniformity of the resistivity profile, but for shallow depth, a small transient electromagnetic system might be reasonable ${ }^{[5]}$. The late time domain corresponds to the deep underground depth, and the prospecting,such as Soundings ${ }^{[6]}$ or coal mine goaf ${ }^{7]}$, has achieved good results.

In this study, a small system of transient electromagnetic of magnetic disource is selected, which provides the possibility to identify the resistivity of shallow media.The multi-offset method, which does not frequently move artificial magnetic field source, can greatly improve the efficiency of field work. The optimal calculation method of apparent resistivity in one dimension full time domain eliminates the subjective factor of dividing time domain and achieves the purpose of detecting different depth at the same time.

\section{The fundamental theory}

As shown in Fig. 1 and the followings, the expression of the magnetic field component of the half-space TEM is selected and we establish the working model of multioffset magnetic disource TEM. The small circular coils regarded as magnetic dipoles serve as artificial field sources and the small coils at different offsets serve as the receiving coils.

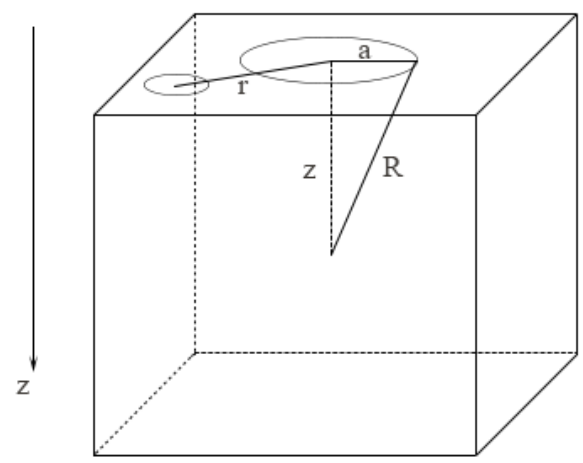

Fig. 1. Working model of multi-offset magnetic disource TEM.

$$
\begin{gathered}
B_{z}(t)=-\frac{\mu_{0} I N S}{4 \pi r^{5}}\left[1-\left(1-\frac{9}{u^{2}}\right) \Phi(u)-\sqrt{\frac{2}{\pi}} e^{-u^{2} / 2}\left(\frac{9}{u}+2 u\right)\right] \\
u=\sqrt{\frac{\mu \sigma}{2 t}} R \\
\Phi(u)=\sqrt{\frac{2}{\pi}} \int_{0}^{u} e^{-\frac{t^{2}}{2}} d t \\
R=\sqrt{a^{2}+z^{2}} \\
S=\pi a^{2}
\end{gathered}
$$

\footnotetext{
* Corresponding author: moskit850@aliyun.com
} 
$\mu_{0}$ is vacuum magnetic permeability, $I$ is magnitude of current, $N$ is the coil number of turns, $S$ is single turn coil area, $\sigma$ is electrical conductivity $\Phi(u)$ is a probabilistic integral function, $t$ is the moment, $a$ is circular coil radius and $z$ is the depth.

\section{Calculation and disscussion}

In this chapter, the control variable method will serve to carry out one-dimensional forward modeling and optimization calculation for the three variables of the moment, offset and apparent resistivity, then we shall discuss the applicable scope of each variable.

After eliminating the point of magnitude difference between the data and the adjacent data, the following pictures are obtained in this chapter.

\subsection{Magnetic dipole source TEM one-dimension forward modeling}

First, the control variable method was used to change the three variables of the moment, offset and apparent resistivity, and then the corresponding magnetic field components were calculated, trying to understand the influence of the changes of each variable on the calculation results of magnetic field components.

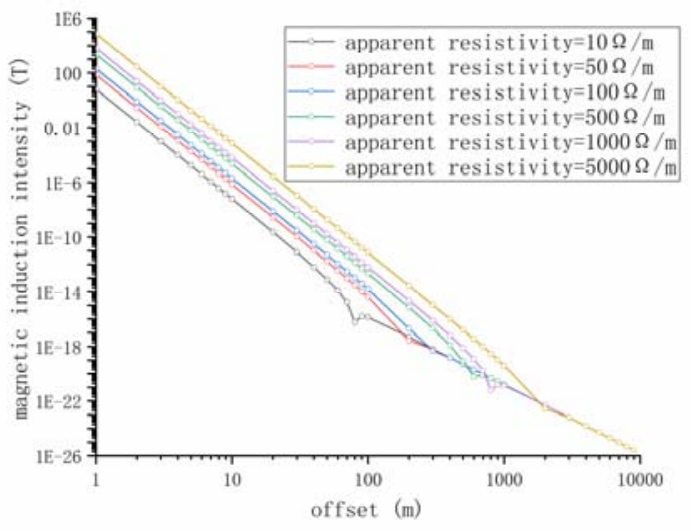

Fig. 2. These data are the only changing the offset $r$ forward modeling results at the moment $0.0001 \mathrm{~s}$, respectively, under apparent resistivity $10,50,100,500,1000,5000 \Omega / \mathrm{m}$ conditions.

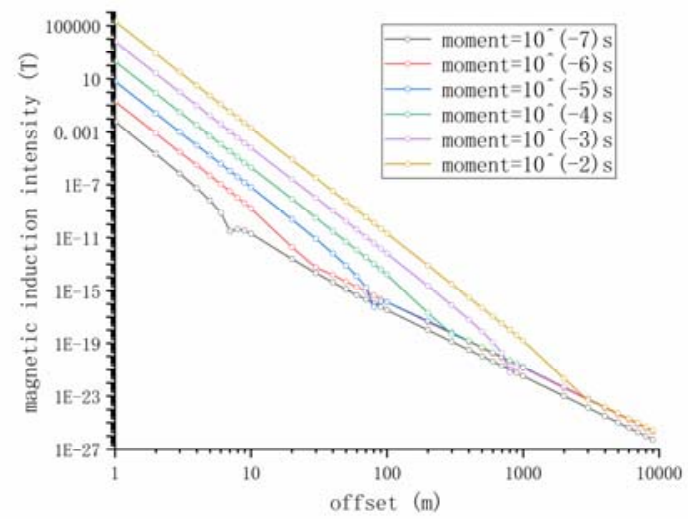

Fig. 3. These data are the only changing the offset $r$ forward modeling results at the apparent resistivity $100 \Omega / \mathrm{m}$, respectively, under moment $10^{-7}, 10^{-6}, 10^{-}$ ${ }^{5}, 10^{-4}, 10^{-3}$ and $10^{-2} \mathrm{~s}$ conditions.

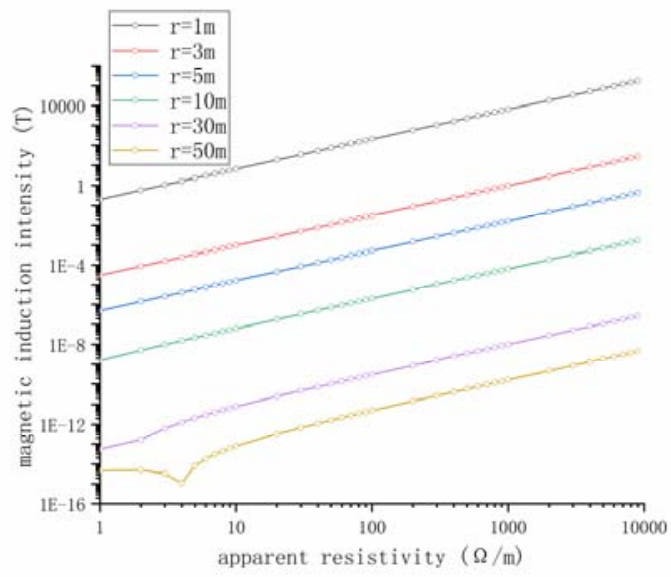

Fig. 4. These data are the only changing apparent resistivity forward modeling results at the moment $0.0001 \mathrm{~s}$, respectively, under the offset $1,3,5,10,30$ and $50 \mathrm{~m}$ conditions.

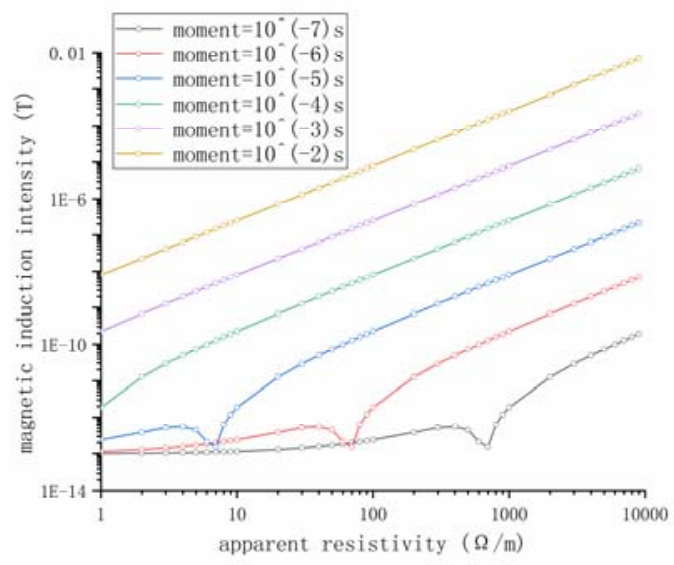

Fig. 5. These data are the only changing apparent resistivity forward modeling results at offset $20 \mathrm{~m}$, respectively, under moment $10^{-7}, 10^{-6}, 10^{-5}, 10^{-4}, 10^{-3}$ and $10^{-2}$ s conditions.

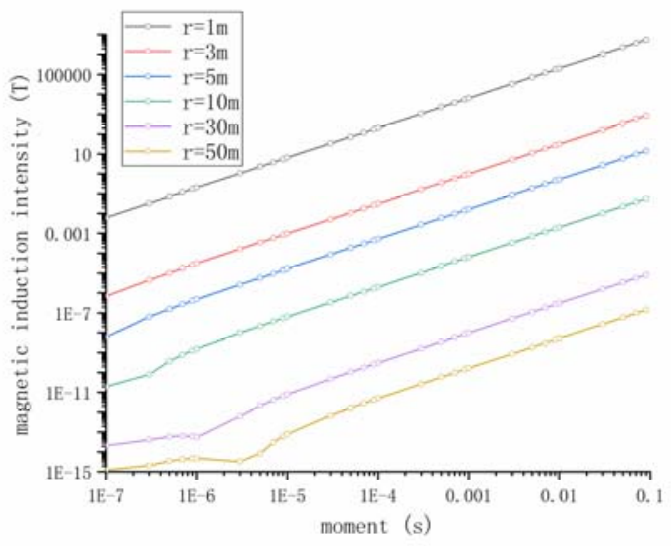

Fig. 6. These data are the only changing the moment forward modeling results at the apparent resistivity 
$100 \Omega / \mathrm{m}$, respectively, under the offset $1,3,5,10,30$ and $50 \mathrm{~m}$ conditions.

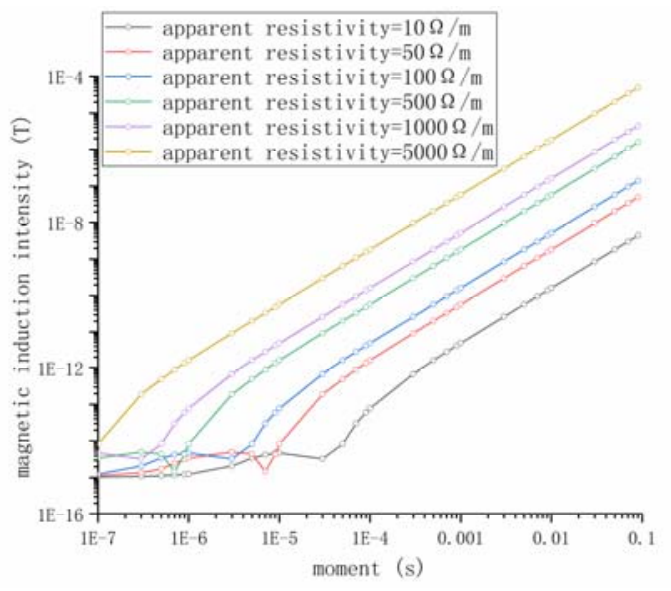

Fig. 7. These data are the only changing the moment forward modeling results at the offset $50 \mathrm{~m}$, respectively, under apparent resistivity $10,50,100,500,1000,5000 \Omega / \mathrm{m}$ conditions.

According to the above picture, we analyze that, for a small system, such as multi-offset magnetic disource TEM, conservatively speaking, the data of $10^{-7}$ and $10^{-6} \mathrm{~s}$ at moments close to the cut-off time of artificial magnetic field source is obviously not continuous. It is difficult to distinguish different resistivities when the migration distance is over $100 \mathrm{~m}$, and the reasonable range of the migration distance is basically $1 \sim 100 \mathrm{~m}$. The apparent resistivity recognition ability of shallow media from $10^{-7}$ to $10^{-6} \mathrm{~s}$ is weak, but in $10^{-6} \sim 10^{-2} \mathrm{~s}$, offset in $1 \sim 100 \mathrm{~m}$ and apparent resistivity in $100 \sim 5000 \Omega / \mathrm{m}$ scope of the three constraints, can always get good results.

\section{2 magnetic dipole source TEM one-dimension optimization calculation}

Equation (6) is Halley's method ${ }^{[8]}$ expression which is used to iterative calculation in the magnetic field component in the moment, offset and apparent resistivity of the three variables.

$$
x_{n+1}=x_{n}-\frac{B_{z}\left(x_{n}\right)}{B_{z}^{\prime}\left(x_{n}\right)-\frac{B_{z}\left(x_{n}\right) B_{z}^{\prime \prime}\left(x_{n}\right)}{2 B_{z}^{\prime}\left(x_{n}\right)}}
$$

In Equation (6), $B_{z}$ is the magnetic field component, $B_{z}$ 'is the first derivative of $B_{z}$ and $B_{z}$ "is the second derivative of $B_{z}$. Because of the use of control variable method, $x_{n}$ is one of the three variables mentioned above in each calculation. Each data point in this section is iterated no more than 20 times, trying to calculate the result.

The optimization calculation process is aimed to simulate the one-dimensional inversion work after field acquisition of magnetic field component data, so that it would be easier to analyze the advantages and disadvantages of multi-offset magnetic disource TEM.

The results of simulated optimization calculation with known magnetic field components show that the moment, offset or apparent resistivity can be accurately solved in most time domain of transient electromagnetic fields, and it is feasible to theoretically calculate the apparent resistivity in the all-time domain. The method may have a large deviation for the calculation of shallow low resistance bodies. The results show that the smaller the offset is, the more accurate the results are. It is considered that it is difficult for a magnetic dipole source to produce a strong magnetic field with a wide range of influence. Therefore, it is suggested to carry out actual field work and try to work within the offset range of $100 \mathrm{~m}$, so as to increase the number of turns of the coil to make up for the shortage of magnetic field component strength.

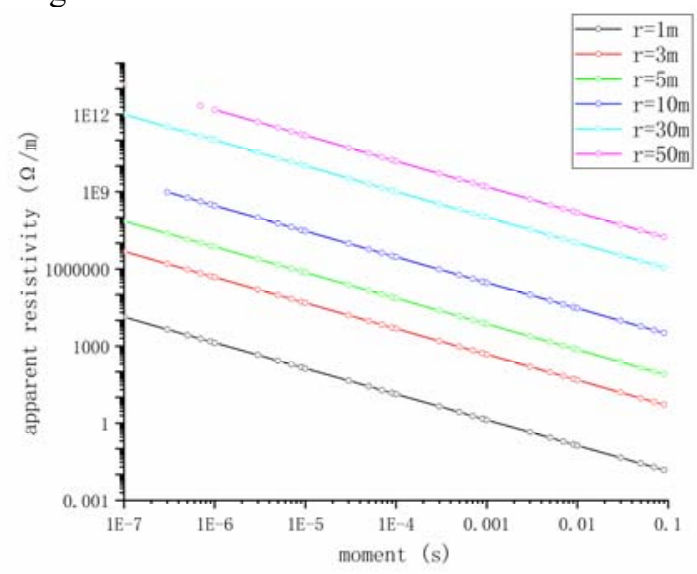

Fig. 8. These data are used to assume that the measured magnetic induction intensity is $\mathrm{B}=10 \mathrm{~T}$. Under the condition of offset $r=1,3,5,10,30$ and $50 \mathrm{~m}$, and then the optimal calculation result of apparent resistivity would be iteratively got.

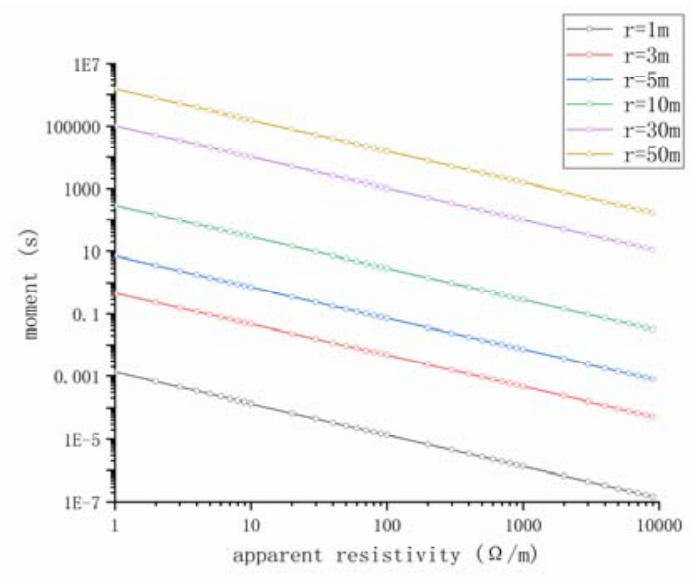

Fig. 9. These data are used to assume that the measured magnetic induction intensity is $\mathrm{B}=10 \mathrm{~T}$. Under the condition of offset $r=1,3,5,10,30$ and $50 \mathrm{~m}$, respectively, and then the optimal calculation result of moment would be iteratively got. 


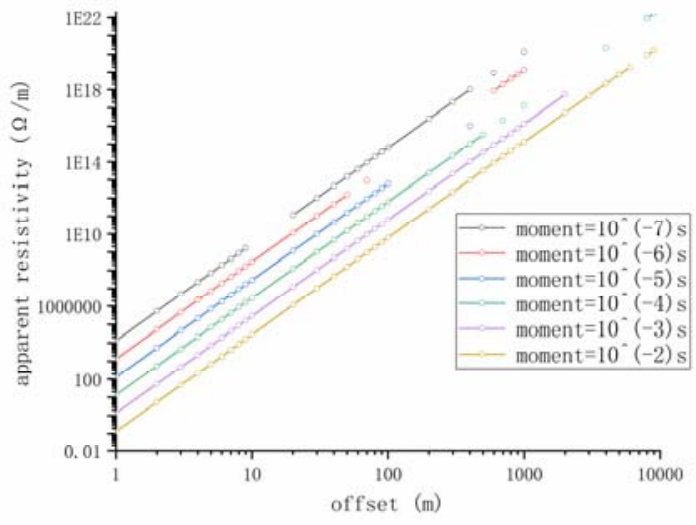

Fig. 10. These data are used to assume that the measured magnetic induction intensity is $\mathrm{B}=10 \mathrm{~T}$. Under the condition of moment $10^{-7}, 10^{-6}, 10^{-5}, 10^{-4}, 10^{-3}$ and $10^{-}$ ${ }^{2} \mathrm{~S}$, respectively, and then the optimal calculation result of apparent resistivity would be iteratively got.

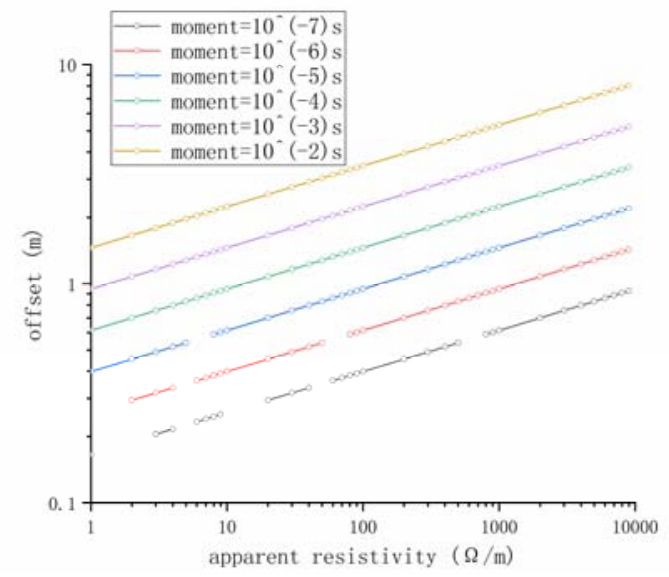

Fig. 11. These data are used to assume that the measured magnetic induction intensity is $\mathrm{B}=10 \mathrm{~T}$. Under the condition of moment $10^{-7}, 10^{-6}, 10^{-5}, 10^{-4}, 10^{-3}$ and $10^{-}$ ${ }^{2} \mathrm{~S}$, respectively, only the offset is changed, and then the optimal calculation result of offset would be iteratively got.

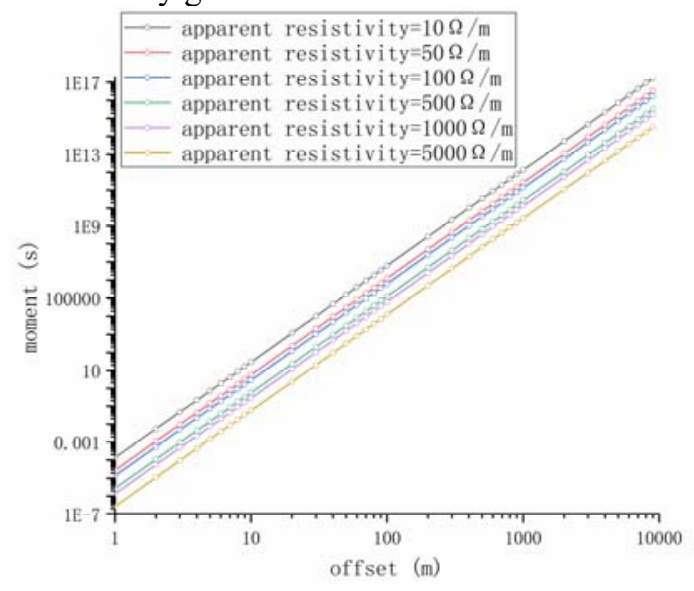

Fig. 12. These data are used to assume that the measured magnetic induction intensity is $\mathrm{B}=10 \mathrm{~T}$. Under the condition of apparent resistivity $10,50,100,500,1000,5000 \Omega / \mathrm{m}$, respectively, and then the optimal calculation result of moment would be iteratively got.

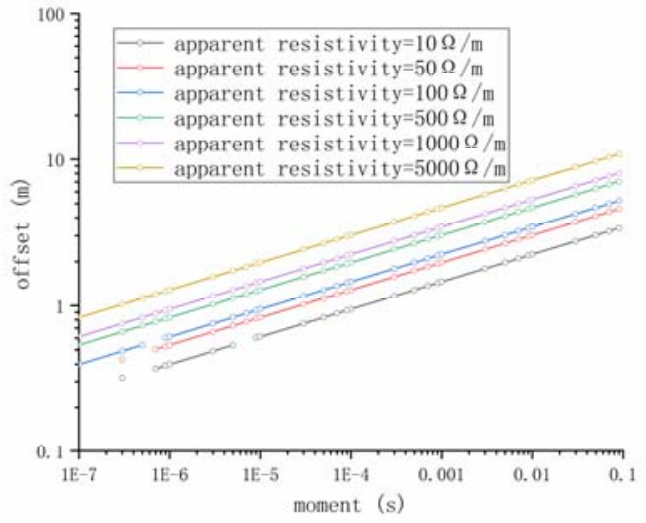

Fig. 13. These data are used to assume that the measured magnetic induction intensity is $B=10 \mathrm{~T}$. Under the condition of apparent resistivity $10,50,100,500,1000,5000 \Omega / \mathrm{m}$, respectively, and then the optimal calculation result of offset would be iteratively got.

\section{Conclusions}

In this paper, it is proved that the multi-offset MTEM is feasible to calculate the apparent resistivity in the full time domain theoretically, avoiding the problem of artificially dividing the early, middle and late time domains.

Optimization results show Halley's method can quickly converge iteration, which greatly speeds up the computing efficiency; It may be difficult to detect the shallow layer of the near surface when the time domain value is very small, especially the low-resistivity medium of the near surface; This kind of small transient electromagnetic system requires a higher range of offset distance, and the closer the distance is to the magnetic dipole source, the more accurate the optimization calculation result will be.

\section{Acknowledgement}

The project was funded by the Natural Science Foundation of the Hebei Province (No. D2018403063).

\section{References}

1. Yansong Hao, Bo $\mathrm{Hu}$, Runqiao $\mathrm{Yu}$, Lijia $\mathrm{Wu}$. GEOPHYSICAL \& GEOCHEMICAL EXPLORATION. 362 (2012), pp1034-1039.

2. Haiyan Yang, Jianhua Yue, Fengping Li. CHINESE JOURNAL OF GEOPHYSICS. 629 (2019), 36153628.

3. Juan Antonio Torres-Martinez, Abrahan Mora, José Alfredo Ramos-Leal, Janete Morán-Ramírez, Claudia Arango-Galván, Jürgen Mahlknecht. Hydrogeology Journal. 27, (2020), pp2955-2972.

4. Rani Payal,Soupios Pantelis,Barsukov. Journal of Applied Geophysics, 176, (2020), pp1-12. 
5. Plotnikov A.E. Russian Geology and Geophysics, 557 (2014), pp907-914.

6. P. O. Barsukov, E. B. Fainberg. Izvestiya, Physics of the Solid Earth, 542 (2018), pp349-358.

7. Jianghao Chang, Jingcun $\mathrm{Yu}$, Zhixin Liu. 133 (2016), pp539-550.

8. Qingxin Meng, Xiangyun Hu, Heping Pan, Yufei Xi. Journal of Applied Geophysics. 151 (2018), pp328342 . 